\title{
Review of $\phi_{3}$ measurements at Belle
}

\author{
Daniel Červenkov ${ }^{1, a}$ on behalf of the Belle collaboration
}

${ }^{1}$ Faculty of Mathematics and Physics, Charles University in Prague, Czech Republic

\begin{abstract}
One of the main goals of the Belle experiment (and the upcoming Belle II experiment) is to measure parameters of electro-weak $\mathrm{CP}$ violation (CPV). A useful way to parametrize $\mathrm{CPV}$ is through the use of unitarity triangles. A brief introduction to unitarity triangle measurements of $\mathrm{CP}$ violation is presented, followed by a summary of recent results from the Belle experiment concerning measurements of the least well constrained angle of the standard unitarity triangle $-\phi_{3} / \gamma$.
\end{abstract}

\section{Unitarity Triangle}

Charge-parity (CP) symmetry in weak interactions is broken by a single irreducible complex phase in the Cabibbo-Kobayashi-Maskawa (CKM) quark mixing matrix [1], [2]. This matrix is embedded in the charged current Lagrangian

$$
\mathcal{L}_{\mathrm{CC}}=\frac{g}{2 \sqrt{2}}(\bar{u} \bar{c} \bar{t}) \gamma^{\mu}\left(1-\gamma_{5}\right)\left(\begin{array}{ccc}
V_{u d} & V_{u s} & V_{u b} \\
V_{c d} & V_{c s} & V_{c b} \\
V_{t d} & V_{t s} & V_{t b}
\end{array}\right)\left(\begin{array}{l}
d \\
s \\
b
\end{array}\right) W_{\mu}^{+}+\text {h.c. . }
$$

It is useful to introduce unitarity triangles in order to parametrize the CKM matrix. The matrix is unitary, which can be written down in the following way,

$$
\left(\begin{array}{ccc}
V_{u d}^{*} & V_{c d}^{*} & V_{t d}^{*} \\
V_{u s}^{*} & V_{c s}^{*} & V_{t s}^{*} \\
\mathbf{V}_{\mathbf{u b}}^{*} & \mathbf{V}_{\mathbf{c b}}^{*} & \mathbf{V}_{\mathbf{t b}}^{*}
\end{array}\right)\left(\begin{array}{lll}
\mathbf{V}_{\mathbf{u d}} & V_{u s} & V_{u b} \\
\mathbf{V}_{\mathbf{c d}} & V_{c s} & V_{c b} \\
\mathbf{V}_{\mathbf{t d}} & V_{t s} & V_{t b}
\end{array}\right)=\left(\begin{array}{ccc}
1 & 0 & 0 \\
0 & 1 & 0 \\
\mathbf{0} & 0 & 1
\end{array}\right)
$$

Eq. 2 is equivalent to 9 complex scalar equations. Let us focus on the ones that are equal to 0 . Out of these, only 3 are independent. We can write down, e.g., the equation comprised of the terms marked in bold.

$$
V_{u d} V_{u b}^{*}+V_{c d} V_{c b}^{*}+V_{t d} V_{t b}^{*}=0 .
$$

This is a sum of 3 complex numbers that is equal to $0-$ any such equation represents a triangle in the complex plane (however, the triangle might be degenerate - have no area).

The triangle we picked is the standard choice, as it has the benefit of its angles being of the same order. This in turn means, they can be measured independently with reasonable relative errors. Furthermore, the triangle is usually rotated so one of its sides lies on the real axis and it is normalized to a unit length, as can be seen in Fig. 1.

\footnotetext{
ae-mail: cervenkov@ipnp.mff.cuni.cz
} 


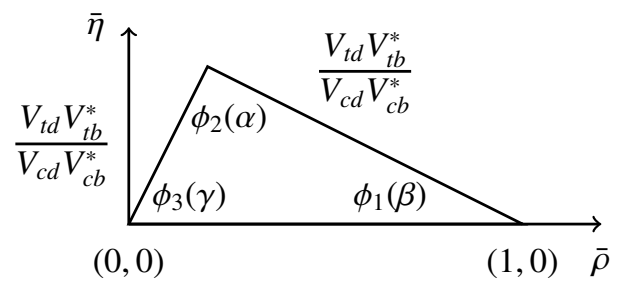

Figure 1. The standard unitarity triangle (Belle angle naming convention first, BaBar convention in parentheses).

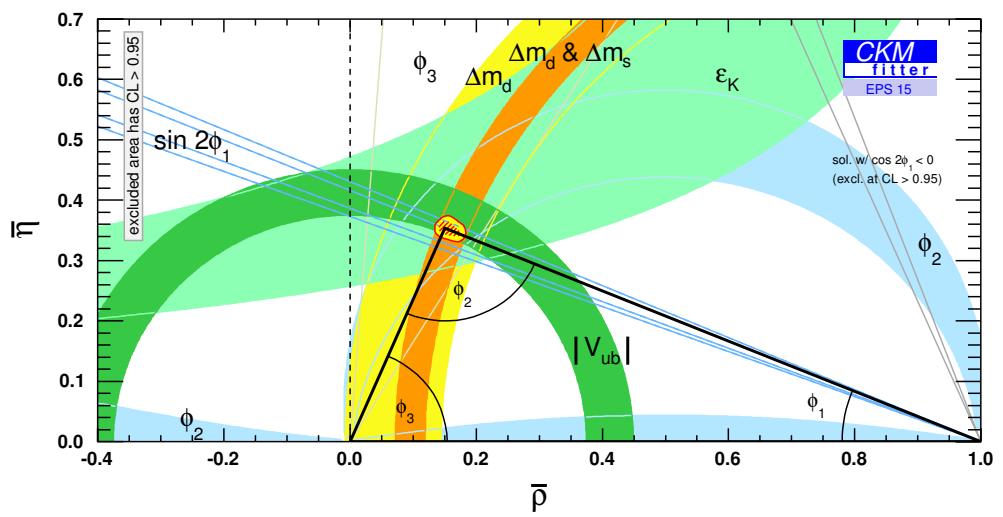

Figure 2. CKMfitter group's global unitarity triangle fit.

The fact, that there is a non-trivial, non-degenerate unitarity triangle implies CP violation. Moreover, careful measurement of the triangle can reveal beyond Standard Model (BSM) physics. Telltale BSM signs would be if the triangle is actually not a triangle (its sides don't close), or if there is a discrepancy between measurements from tree-dominated processes and loop-dominated processes.

The various CKM measurements are combined into a single plot by the CKMfitter group [3], shown in Fig. 2. It's also worth noting that $\phi_{3}$ is the least well constrained angle of the unitarity triangle.

\section{Methods for Measuring $\phi_{3}$}

The most powerful and theoretically clean way of measuring $\phi_{3}$ is based on the interference between $b \rightarrow \bar{u} c s$ and $b \rightarrow u \bar{c} s$ tree level amplitudes [4]. What we mean by saying theoretically clean is that there is no penguin graph contribution to these processes and consequently no theoretical uncertainty connected to the hadronic parameters, as they can be obtained from experiment. It is worth noting that $\phi_{3}$ is the only parameter of CP violation that can be measured solely from tree-level processes. However, $\phi_{3}$ measurement precision is limited by small branching fractions of the involved processes.

An example of the mentioned type of processes is $B^{ \pm} \rightarrow D^{(*)} K^{ \pm}$followed by $D \rightarrow f$, and $B^{ \pm} \rightarrow$ $\bar{D}^{(*)} K^{ \pm}$followed by $\bar{D} \rightarrow f$, where $f$ is a common final state. Interference between these two paths gives rise to direct CP violation. Diagrams of the relevant decays are depicted in Fig. 3. 

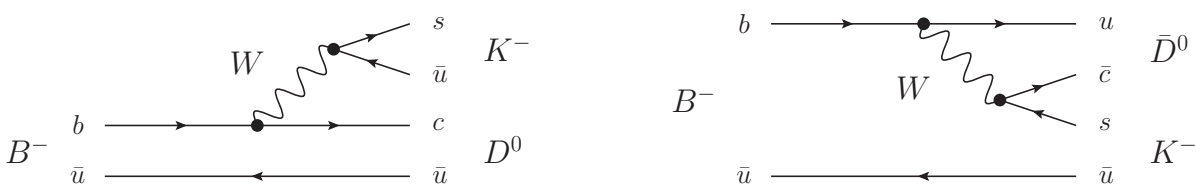

Figure 3. Diagrams of the two tree-level $B^{-} \rightarrow D K^{-}$decays.

Most analyses neglect the effects of neutral $D$ meson mixing and $\mathrm{CP}$ violation as these are expected to be small [5]. However, $D$ mixing and $\mathrm{CP}$ violation can be included at no cost to the uncertainty by using measured values from other studies [6].

\subsection{GLW Method}

The method was first proposed by Gronau, London and Wyler [4], [7]. Their idea was that a $B$ meson can decay weakly to a state with a $D^{0}$ or $\bar{D}^{0}$. But if we reconstruct the neutral $D$ meson from a final state that is a CP-eigenstate, we actually select a superposition $\left(D^{0} \pm \bar{D}^{0}\right) / \sqrt{2}$. We label these states $D_{C P^{+}}$and $D_{C P^{-}}$for the CP-even and CP-odd states respectively. We can then construct 4 observables that encode the $\mathrm{CP}$ violation parameters,

$$
\begin{gathered}
\mathcal{R}_{C P^{ \pm}}=2 \frac{\Gamma\left(B^{-} \rightarrow D_{C P^{ \pm}} K^{-}\right)+\Gamma\left(B^{+} \rightarrow D_{C P^{ \pm}} K^{+}\right)}{\Gamma\left(B^{-} \rightarrow D_{f a v} K^{-}\right)+\Gamma\left(B^{+} \rightarrow D_{f a v} K^{+}\right)}=1+r_{B}^{2} \pm 2 r_{B} \cos \left(\delta_{B}\right) \cos \left(\phi_{3}\right), \\
\mathcal{A}_{C P^{ \pm}}=\frac{\Gamma\left(B^{-} \rightarrow D_{C P^{ \pm}} K^{-}\right)-\Gamma\left(B^{+} \rightarrow D_{C P^{ \pm}} K^{+}\right)}{\Gamma\left(B^{-} \rightarrow D_{C P^{ \pm}} K^{-}\right)+\Gamma\left(B^{+} \rightarrow D_{C P^{ \pm}} K^{+}\right)}= \pm r_{B} \sin \left(\delta_{B}\right) \sin \left(\phi_{3}\right) / R_{C P^{ \pm}},
\end{gathered}
$$

where $D_{\text {fav }}$ signifies that the $D$ meson is reconstructed in a favored hadronic mode such as $D^{0} \rightarrow$ $K^{-} \pi^{+}, r_{B}$ is the magnitude of the ratio of $B \rightarrow \bar{D}^{0} K^{-}$and $B \rightarrow D^{0} K^{-}$amplitudes and $\delta_{B}$ is the strong phase difference between them. $\mathcal{A}_{C P^{ \pm}}$different from 0 means $\mathrm{CP}$ is violated in these processes.

Notice that the system is not over-constrained by the 4 observables even though there are 3 parametes, as $\mathcal{R}_{C P^{+}} \mathcal{A}_{C P^{+}}=-\mathcal{R}_{C P^{-}} \mathcal{A}_{C P^{-}}$.

\subsection{ADS Method}

Atwood, Dunietz and Soni [8] realized that CKM-suppressed decays can also be used to measure $\phi_{3}$. Let us consider the process $B^{-} \rightarrow\left[K^{+} \pi^{-}\right]_{D} K^{-}$, where the parentheses represent a final state that was produced from an intermediate $D$ resonance. The full final state can be reached in two ways either CKM-favored $B^{-} \rightarrow D^{0} K^{-}$followed by CKM-suppressed $D^{0} \rightarrow K^{+} \pi^{-}$, or CKM-suppressed $B^{-} \rightarrow \bar{D}^{0} K^{-}$followed by CKM-favoured $D^{0} \rightarrow K^{-} \pi^{+}$.

In contrast to the GLW method, when the $D$ meson decays to a non-CP-eigenstate, one has to factor in the magnitude of the ratio of the suppressed and favored $D$ decays $r_{D}$ as well as their relative strong phase $\delta_{D}$, much like we did for the $B$ decay. Fortunately, these hadronic parameters can be obtained from mixing measurements [9].

The ADS observables are similar to the GLW ones, however there are only 2 of them as they are charge-averaged, 


$$
\begin{aligned}
\mathcal{R}_{A D S} & =\frac{\Gamma\left(B^{-} \rightarrow\left[K^{+} \pi^{-}\right]_{D} K^{-}\right)+\Gamma\left(B^{+} \rightarrow\left[K^{-} \pi^{+}\right]_{D} K^{+}\right)}{\Gamma\left(B^{-} \rightarrow\left[K^{-} \pi^{+}\right]_{D} K^{-}\right)+\Gamma\left(B^{+} \rightarrow\left[K^{+} \pi^{-}\right]_{D} K^{+}\right)} \\
& =r_{B}^{2}+r_{D}^{2}+2 r_{B} r_{D} \cos \left(\delta_{B}+\delta_{D}\right) \cos \left(\phi_{3}\right) . \\
\mathcal{A}_{A D S} & =\frac{\Gamma\left(B^{-} \rightarrow\left[K^{+} \pi^{-}\right]_{D} K^{-}\right)-\Gamma\left(B^{+} \rightarrow\left[K^{-} \pi^{+}\right]_{D} K^{+}\right)}{\Gamma\left(B^{-} \rightarrow\left[K^{+} \pi^{-}\right]_{D} K^{-}\right)+\Gamma\left(B^{+} \rightarrow\left[K^{-} \pi^{+}\right]_{D} K^{+}\right)} \\
& =2 r_{B} r_{D} \sin \left(\delta_{B}+\delta_{D}\right) \sin \left(\phi_{3}\right) / \mathcal{R}_{A D S} .
\end{aligned}
$$

\subsection{Dalitz Plot Method (GGSZ)}

The Dalitz plot analysis method for $\phi_{3}$ measurements was proposed by Giri, Grossman, Soffer and Zupan [10] and independently by Bondar [11]. The idea behind it is to use (usually self-conjugate) multi-body final states accessible to both $D^{0}$ and $\bar{D}^{0}$ mesons. One then measures relative phases and magnitudes of their amplitudes for $D$ mesons coming from $B$ decays such as $B^{ \pm} \rightarrow D K^{ \pm}$.

Let us consider a $B^{ \pm} \rightarrow\left[K_{S}^{0} \pi^{+} \pi^{-}\right]_{D} K^{ \pm}$process. Its amplitude can be written down as

$$
\begin{aligned}
& A_{B^{+}}\left(s_{+}, s_{-}\right)=\bar{A}_{D}\left(s_{+}, s_{-}\right)+r_{B} e^{i\left(\delta_{B}+\phi_{3}\right)} A_{D}\left(s_{+}, s_{-}\right), \\
& A_{B^{-}}\left(s_{+}, s_{-}\right)=A_{D}\left(s_{+}, s_{-}\right)+r_{B} e^{i\left(\delta_{B}+\phi_{3}\right)} \bar{A}_{D}\left(s_{+}, s_{-}\right),
\end{aligned}
$$

where we have introduced the Dalitz variables $s_{ \pm}=m_{K_{S}^{0} \pi^{ \pm}}^{2}$ and $A_{D}$ is the amplitude of the $D$ decay. The strong phase $\delta_{B} \equiv \delta_{B}\left(s_{+}, s_{-}\right)$has to have a large variation over the Dalitz plot; if it were constant, there would be no $\phi_{3}$ sensitivity.

Two approaches are possible - model independent, binned analysis (as proposed in the original paper) and model dependent, unbinned analysis. The former divides the Dalitz plot into bins across which there is a small strong phase variation. The events in one bin are then treated equally. Extra input in the form of strong phase measurements from charm factories are required for this method [12].

The second method employs a certain model of the strong phase distribution function across the Dalitz phase-space. While introducing an obvious model uncertainty, it has a higher statistical power, which can be very desirable in low yield analyses.

\subsection{Continuum Suppression}

A vital procedure in these measurements is called continuum suppression. It distinguishes $B$ meson decay events from $e^{+} e^{-} \rightarrow q \bar{q}$ type events, where $q$ stands for $u, d, s$ or $c$ type quarks. The name continuum comes from the fact, that these events hadn't gone through an intermediate $\Upsilon(4 S)$ resonance, in contrast to the $B$ meson decay events.

These backgrounds can be quite prominent, but have very different event shapes (solid angle distribution of decay particles); see Fig. 4. This allows us to exploit a variety of observables such as thrust, sphericity, Fox-Wolfram moments, $\Delta z$ and flavor tag to discriminate against these backgrounds. Usually neural networks or boosted decision trees are used to combine many of the listed variables into a single one with high discriminating power.

\section{Measurements}

All the listed analyses use the entire $711 \mathrm{fb}^{-1}$ Belle data sample containing $772 \times 10^{6} B \bar{B}$ events. Listed uncertainties are statistical first, systematic second. 

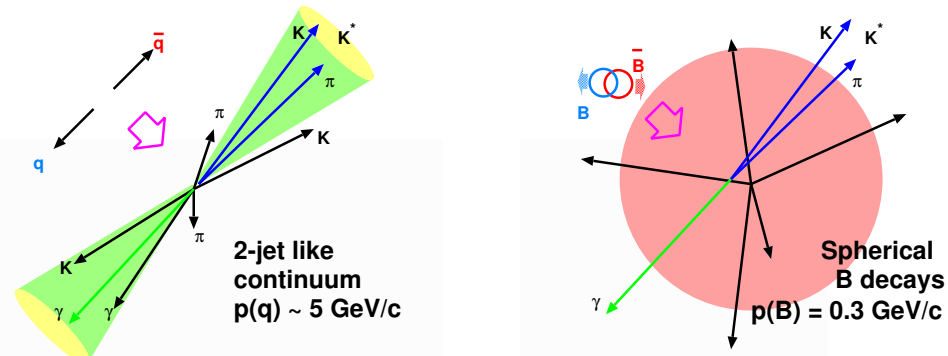

Figure 4. Event shapes for continuum (left) and $B$ decay events (right) [13].

\section{1 $B^{-} \rightarrow D^{* 0} K^{-}, D^{* 0} \rightarrow D^{0} \pi^{0}, D^{0} \rightarrow K_{S}^{0} \pi^{0}, K_{S}^{0} \eta$ (GLW)}

It is of note, that this measurement is a B-factory exclusive - it would be very difficult to study this channel on hadron machines, because of the multiple neutral final-state particles.

The analysis was carried out in both $D^{* 0} \rightarrow D^{0} \pi^{0}$ and $D^{* 0} \rightarrow D^{0} \gamma$ modes. Combining them with CP-even $D^{0}$ final-states $\pi^{+} \pi^{-}$and $K^{+} K^{-}$, yields the following results [14],

$$
\begin{aligned}
& R_{C P+}=+1.19 \pm 0.13 \pm 0.03, \\
& R_{C P-}=+1.03 \pm 0.13 \pm 0.03, \\
& A_{C P+}=-0.14 \pm 0.10 \pm 0.01, \\
& A_{C P-}=+0.22 \pm 0.11 \pm 0.01 .
\end{aligned}
$$

The observables show clear hints of CP asymmetry.

\section{$3.2 B^{-} \rightarrow D^{0} K^{-}, D^{0} \rightarrow K^{+} \pi^{-}$(ADS)}

The authors updated this study with the full Belle dataset, adding $20 \%$ more data to the previous study [15]. They also incorporated several improvements such as a 2D fit for signal yield extraction and a neural network for background suppression. This resulted in a first observation of the suppressed mode, with a $4.1 \sigma$ significance (including systematic uncertainties) [16],

$$
\begin{aligned}
& R_{D K}=\left(1.63_{-0.41-0.13}^{+0.44+0.07}\right) \times 10^{-2}, \\
& A_{D K}=-0.39_{-0.28-0.13}^{+0.26+0.04} .
\end{aligned}
$$

3.3 $B^{0} \rightarrow D^{0} K^{* 0}, K^{* 0} \rightarrow K^{+} \pi^{-}, D^{0} \rightarrow K^{-} \pi^{+}$(ADS)

A major advantage of this analysis is that it uses a self-tagging channel, because of the $K^{*}$ decay. This in turn boosts efficiency. While the study uses the ADS method, the fact that $K^{*}$ has a natural width larger than the experimental resolution, leads to some complications which require a modified definition of the ADS observables. Further details can be found in [17]. The obtained result is [18]

$$
R_{D K^{* 0}}=\left(4.1_{-5.0-1.8}^{+5.6+2.8}\right) \times 10^{-2} .
$$

As the $R_{D K^{* 0}}$ value is not significant, an upper limit was established

$$
R_{D K^{* 0}}<0.16 \quad \text { (95\% C.L.). }
$$




\section{$3.4 B^{-} \rightarrow D^{0} K^{-}, D^{0} \rightarrow K^{+} \pi^{-} \pi^{0}$ (ADS)}

The fact that there is a 3-body final-state in this ADS analysis means that the strong phase difference between the interfering processes can vary over the phase-space. This can "dilute" direct CP violation effects. To quantify this dilution, correlated $D \bar{D}$ production is used [19], [20]. Fortunately, the effect is small in this channel and allows for a $\mathrm{CP}$ violation measurement [21]:

$$
\begin{aligned}
& R_{D K}=(1.98 \pm 0.62 \pm 0.24) \times 10^{-2} \\
& A_{D K}=0.41 \pm 0.30 \pm 0.05
\end{aligned}
$$

This is a first evidence for the suppressed decay, with a $3.2 \sigma$ significance.

$$
\text { 3.5 } B^{-} \rightarrow D^{0} K^{-}, D^{0} \rightarrow K_{S}^{0} \pi^{+} \pi^{-} \text {(Dalitz) }
$$

This analysis is the first model-independent measurement of $\phi_{3}$ in the relevant channel. The authors report a result of [22]

$$
\phi_{3}=\left(77_{-14.9}^{+15.1} \pm 4.1 \pm 4.3\right)^{\circ},
$$

where the third uncertainty comes from the precision of the strong-phase parameters obtained by CLEO, which are an external input to this analysis.

This uncertainty is comparable to the model uncertainties from the latest Belle and BaBar measurements: $3^{\circ}-9^{\circ}$. For future experiments, the model uncertainty is expected to dominate as there will be more statistics and, possibly, better systematics control. On the other hand, the precision of the strong-phase parameters measurement will improve as BES-III results supersede CLEO's.

\section{6 $B^{0} \rightarrow D^{0} K^{* 0}, K^{* 0} \rightarrow K^{+} \pi^{-}, D^{0} \rightarrow K_{S}^{0} \pi^{+} \pi^{-}$(Dalitz)}

This analysis also uses the model-independent approach and resulted in establishing an upper limit on the suppressed vs. favored ratio $r_{S}<0.87$ at the $68 \%$ confidence level [23]. This value is closely related to $\phi_{3}$ sensitivity because the statistical uncertainty of $\phi_{3}$ measurements is proportional to $1 / r_{S}$.

The authors also report the "raw" observables $x_{ \pm}, y_{ \pm}$which are defined as

$$
\begin{aligned}
& x_{ \pm}=r_{S} \cos \left(\delta_{S} \pm \phi_{3}\right), \\
& y_{ \pm}=r_{S} \sin \left(\delta_{S} \pm \phi_{3}\right)
\end{aligned}
$$

and are measured to be

$$
\begin{aligned}
& x_{-}=+0.4_{-0.6-0.1}^{+1.0+0.0} \pm 0.0, \\
& y_{-}=-0.6_{-1.0-0.0}^{+0.8+0.1} \pm 0.1, \\
& x_{+}=+0.1_{-0.4-0.1}^{+0.7+0.0} \pm 0.1, \\
& y_{+}=+0.3_{-0.8-0.1}^{+0.5+0.0} \pm 0.1,
\end{aligned}
$$

where the third uncertainty again comes from the precision of the strong-phase parameters, as in Sec. 3.5.

These observables have the benefit, that they can be readily merged with similar results from other studies for a combined measurement. 

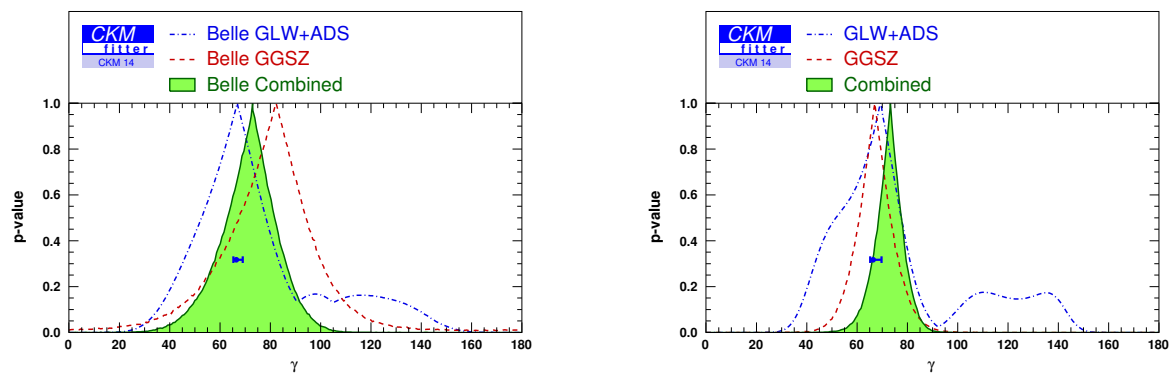

Figure 5. $\phi_{3}$ combination fit; Belle (left) and world (right) [3].

\section{Conclusion}

Precision of the measurements of the $\phi_{3}$ angle of the unitarity triangle is currently dominated by statistical uncertainty. The current Belle and world combination $\phi_{3}$ fits can be seen in Fig. 5.

With the new generation of flavor experiments, Belle II and the LHCb upgrade, along with inputs from charm threshold provided by BES-III, the world combination of the least well-known parameter of the unitarity triangle $-\phi_{3}$, could reach sub-degree precision. It is important we continue constraining the unitarity triangle as New Physics could very well be hiding there.

\section{Acknowledgement}

The author would like to thank the Grant Agency of the Charles University in Prague (GAUK), which supported this work via the grant \#1370313.

\section{References}

[1] N. Cabibbo, Phys. Rev. Lett. 10, 531 (1963)

[2] M. Kobayashi, T. Maskawa, Progr. Theoret. Phys. 49, 652 (1973)

[3] J. Charles et al. (CKMfitter Group), Eur. Phys. J. C41, 1 (2005), hep-ph/0406184

[4] M. Gronau, D. London, Phys. Lett. B253, 483 (1991)

[5] A.F. Falk, Y. Grossman, Z. Ligeti, Y. Nir, A.A. Petrov, Phys. Rev. D69, 114021 (2004), hep-ph/0402204

[6] M. Rama, Phys. Rev. D89, 014021 (2014), 1307.4384

[7] M. Gronau, D. Wyler, Phys. Lett. B265, 172 (1991)

[8] D. Atwood, I. Dunietz, A. Soni, Phys. Rev. D 63, 036005 (2001)

[9] K.A. Olive et al. (Particle Data Group), Chin. Phys. C38, 090001 (2014)

[10] A. Giri, Y. Grossman, A. Soffer, J. Zupan, Phys. Rev. D 68, 054018 (2003)

[11] A. Bondar, unpublished, Belle Dalitz analysis meeting, (2002)

[12] J. Libby et al. (CLEO Collaboration), Phys. Rev. D 82, 112006 (2010)

[13] M. Nakao, unpublished, Belle Analysis School, (2009)

[14] K. Trabelsi, Y. Horii et al. (Belle Collaboration), unpublished, CKM Preliminary, (2012)

[15] Y. Horii, K. Trabelsi, H. Yamamoto et al. (Belle Collaboration), Phys. Rev. D 78, 071901 (2008) 
[16] Y. Horii, K. Trabelsi, H. Yamamoto et al. (Belle Collaboration), Phys. Rev. Lett. 106, 231803 (2011), 1103. 5951

[17] B. Aubert et al. (BaBar Collaboration), Phys. Rev. D 80, 031102 (2009)

[18] K. Negishi, Y. Horii, Y. Onuki, T. Sanuki, H. Yamamoto et al. (Belle Collaboration), Phys. Rev. D86, 011101 (2012), 1205.0422

[19] N. Lowrey et al. (CLEO Collaboration), Phys. Rev. D80, 031105 (2009), 0903. 4853

[20] J. Libby et al., Phys. Lett. B731, 197 (2014), 1401.1904

[21] M. Nayak, J. Libby, K. Trabelsi et al. (Belle Collaboration), Phys. Rev. D88, 091104 (2013), 1310.1741

[22] H. Aihara et al. (Belle Collaboration), Phys. Rev. D85, 112014 (2012), 1204.6561

[23] A. Abdesselam et al. (Belle Collaboration) (2015), 1502.07550 